\title{
Categorization, Designation, and Regionalization of Emergency Care: Definitions, a Conceptual Framework, and Future Challenges
}

Keith E. Kocher, MD, MPH, MPhil, David P. Sklar, MD, Abhishek Mehrotra, MD, Vivek S. Tayal, MD, Marianne Gausche-Hill, MD, and R. Myles Riner, MD

\begin{abstract}
This article reflects the proceedings of a breakout session, "Beyond ED Categorization-Matching Networks to Patient Needs," at the 2010 Academic Emergency Medicine consensus conference, "Beyond Regionalization: Integrated Networks of Emergency Care." It is based on concepts and areas of priority identified and developed by the authors and participants at the conference. The paper first describes definitions fundamental to understanding the categorization, designation, and regionalization of emergency care and then considers a conceptual framework for this process. It also provides a justification for a categorization system being integrated into a regionalized emergency care system. Finally, it discusses potential challenges and barriers to the adoption of a categorization and designation system for emergency care and the opportunities for researchers to study the many issues associated with the implementation of such a system.
\end{abstract}

ACADEMIC EMERGENCY MEDICINE 2010; 17:1306-1311 (c) 2010 by the Society for Academic Emergency Medicine

\section{DEFINITIONS}

$\mathrm{W}$

e begin with definitions of key terms used in discussions of regionalization and categorization of emergency care. Definitions assist in research, planning, and political processes by ensuring that the terms used have the same meaning for administrators, investigators, and policy-makers who may be involved in the planning of integrated networks of emergency care.

\section{Categorization}

Categorization is a process for inventorying, assessing, and cataloguing the emergency care resources, services, capabilities, and capacities of medical care facilities in a community or region, using a criteria-based classification system over a range of emergency care conditions. This process is used to assist physicians, hospitals, health departments, and emergency medical services (EMS) agencies in making informed decisions on how to develop, organize, and appropriately utilize health care resources for the emergency care system. Categorization may be accomplished using self-survey and selfdeclaration by facilities, by external agency survey and verification, or by some combination of the two.

\section{Designation}

Designation is a process for granting a charter as a preferred prehospital receiving facility and/or local or

From the Department of Emergency Medicine, University of Michigan (KEK), Ann Arbor, MI; the Department of Emergency Medicine and the Office of Graduate Medical Education, University of New Mexico (DPS), Albuquerque, NM; the Department of Emergency Medicine, University of North Carolina (AM), Chapel Hill, NC; the Department of Emergency Medicine, Carolinas Medical Center (VST), Charlotte, NC; the Department of Emergency Medicine, Harbor-UCLA Medical Center; Department of Medicine, David Geffen School of Medicine at UCLA (MGH), Torrance, CA; and no affiliation (RMR), Mill Valley, CA.

Breakout session participants: James Amsterdam, Brent Asplin, Carlos A. Camargo, Jr., Kathleen Cowling, Stephen Epstein, Nicole Franks, Angela Gardner, Bobby Heard, Marianne Gausche-Hill, Josh Hilton, Ben Honigman, Greg Hufstetler, Ray Johnson, Keith E. Kocher, Abhishek Mehrotra, John Moorhead, Josh Moskovitz, Terry Mullins, Bryn Mumma, Vivek S. Tayal, Mike Richards, R. Myles Riner, Alex Rosenau, Nels D. Sannddal, Sara Schneider, Philip Scott, Suzanne Shepherd, David P. Sklar, Robert Solomon, Sal Vicario, Arlo Weltge, and Micheal Willy.

Received July 22, 2010; revisions received August 23 and September 2, 2010; accepted September 13, 2010.

Supervising Editor: Robert T. Gerhardt, MD, MPH.

Address for correspondence and reprints: Keith E. Kocher MD; e-mail: kkocher@umich.edu. 
regional referral facility for a certain medical condition or group of related conditions. The fulfillment of this charter is predicated on meeting and maintaining certain capacity, capability, and performance standards and on the commitment of the facility to continually improve the care of patients with these medical conditions. This process is usually implemented by a governmental organization responsible for local or regional planning and oversight of EMS, and may entail funding from a governmental body.

\section{Accreditation}

Accreditation is the process that leads to attestation by an outside agency that a practice, laboratory, department, organization, or institution has met certain predetermined and generally agreed-upon standards. These standards may involve considerations of the type and quality of care, safety, efficiency, cost-effectiveness, and accessibility.

\section{Regionalization}

Regionalization is the matching of medical resources to patient needs to maximize health benefits and outcomes while minimizing cost and use of resources over a specified geographic area. In general, this process implies a level of organization beyond the local level, but below the national level.

\section{Verification}

The act of reviewing, assessing, inspecting, or testing to establish that a service or system meets predetermined standards.

\section{Licensure}

The legal permission granted by governmental bodies to provide specified goods and services.

\section{A CONCEPTUAL FRAMEWORK FOR CATEGORIZATION, DESIGNATION, AND REGIONALIZATION}

Already there are existing or developing efforts to regionalize emergency care. Examples include trauma care, $^{1,2}$ stroke care, ${ }^{3,4}$ cardiac care, ${ }^{5,6}$ poisoning, ${ }^{7,8}$ and both pediatric ${ }^{9,10}$ and adult ${ }^{11,12}$ critical care. To better appreciate the strategies involved in this process, we propose the following conceptual framework for understanding the relationships between categorization, designation, and the development of a regionalized network of emergency care.

Categorization should be viewed both as vertical and horizontal sets of capacities that are typically located in a hospital, but may exist in other related configurations (Figures 1 and 2). Vertical categorization criteria are usually specific to a related set of clinical conditions or a particular patient population, which can be termed clinical silos. In contrast, horizontal categorization criteria cover a broad range of clinical conditions and patient populations. The clinical silos in vertical categorization generally have in common that they involve higher-risk, time-sensitive conditions that frequently require the need for specialized knowledge, skills, equipment, and other resources in order to

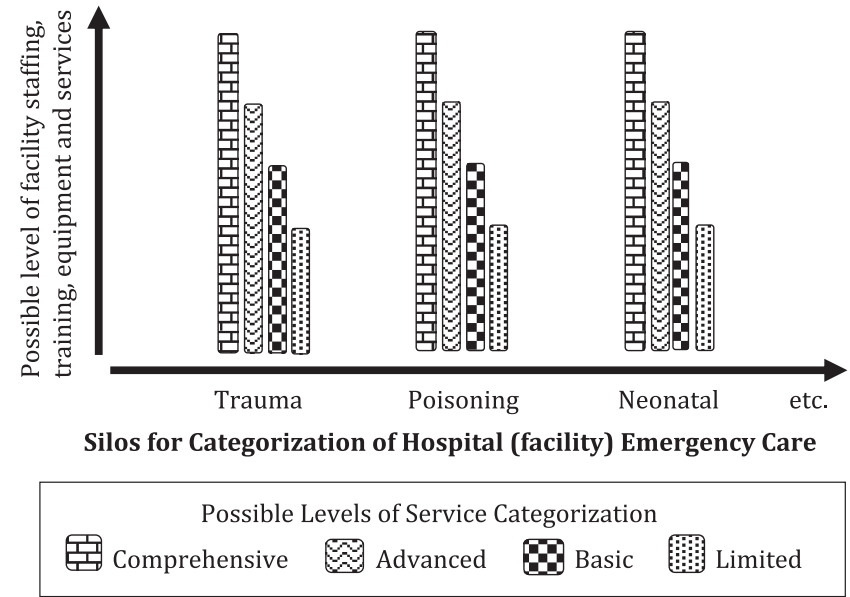

Figure 1. A scheme for the vertical categorization of hospital emergency services.

Comprehensive $=$ comprehensive hospital emergency services for specific disease silos: comprehensive ED and specialized inpatient intensive care and diagnostic, operative, and therapeutic services and equipment and with in-house and/or promptly available physician specialists related to the disease silo. Rarely transfers critically ill patients for poststabilization specialty care. Advanced = advanced hospital emergency services for specific disease silos: advanced ED and inpatient intensive care and most specialized diagnostic, operative, and therapeutic services and equipment and with promptly available physician specialists related to the disease silo. Occasionally transfers critically ill patients for poststabilization specialty care.

Basic = basic hospital emergency services for specific disease silos: basic ED and inpatient care and diagnostic, operative, and therapeutic services and equipment and with selected physician specialists available for consultation. Often transfers some seriously ill and most critically ill patients for stabilization and poststabilization care.

Limited = limited hospital emergency services for specific disease silos: limited ED and inpatient care and diagnostic services and equipment and with limited physician specialists available for consultation. Typically transfers seriously and critically ill patients to higher levels of care for stabilization and poststabilization care.

optimize health outcomes. ${ }^{13}$ These silos may be defined by very narrow clinical conditions, such as emergency care for amputations with the potential for replantation or by very broad sets of medical conditions experienced by a specific patient population, such as emergency care for pediatric patients. Likewise, designation may focus on a very select patient population, as with STelevation myocardial infarction (STEMI) receiving facilities and stroke centers, or on broader patient populations, as with trauma centers and emergency departments (EDs) approved for pediatric emergencies.

Horizontal categorization pertains to ED capabilities and resources available for all types of patients, including those who do not fit into a disease-specific vertical silo. The latter currently make up the majority of those patients arriving at EDs. These include undifferentiated patients with presenting complaints such as abdominal pain, headache, fever, and minor trauma. Critical issues for horizontal categorization include facility space, communication, equipment, diagnostic services, the qualification and availability of ED staff, and the avail- 


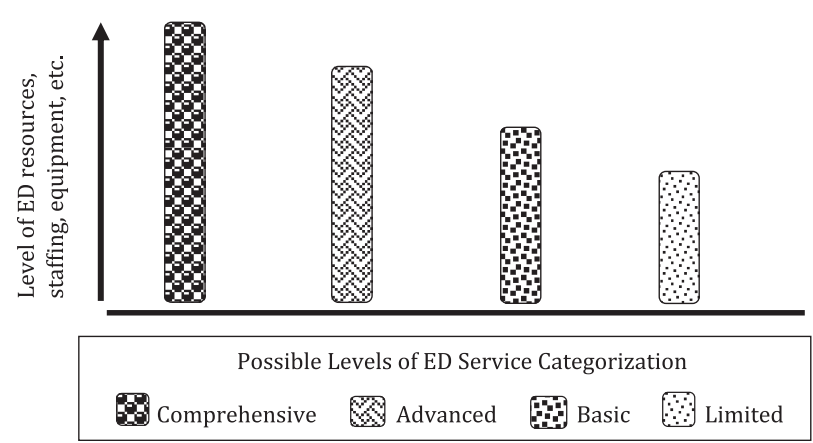

Figure 2. A scheme for the horizontal categorization of hospital ED services.

Comprehensive $=$ comprehensive ED services for a full range of medical conditions, including board-certified emergency physicians on duty $24 / 7$, with access to specialized inpatient intensive care and diagnostic, operative, and therapeutic services and equipment and a wide range of in-house and/or on-call physician specialists. Rarely transfers critically ill patients for poststabilization specialty care.

Advanced $=$ advanced ED services for a broad range of medical conditions, including emergency physicians on duty $24 / 7$, with access to specialized inpatient intensive care and most specialized diagnostic, operative, and therapeutic services and equipment and with promptly available or on-call physician specialists representing most medical and surgical specialties. Occasionally transfers critically ill patients for poststabilization specialty care.

Basic = basic ED services for a moderate range of medical conditions, including emergency physicians on duty $24 / 7$, with access to basic inpatient care and diagnostic, operative, and therapeutic services and equipment and with selected physician specialists on call. Often transfers some seriously ill and most critically ill patients for stabilization and poststabilization care.

Limited = limited ED services for narrow range of medical conditions, including physicians on-call to the ED 24/7, with access to few inpatient care and diagnostic, operative, and therapeutic services and equipment and with limited physician specialists available for consultation. Typically transfers seriously and critically ill patients to higher levels of care for stabilization and poststabilization care.

ability of on-call specialists. Previous assessments of the capabilities for the care of children in general hospital EDs have provided useful methodologies that could be applied to patients of all ages. ${ }^{14,15}$

Inherent to any emergency care categorization scheme is the identification of a hierarchy of expertise and capability, termed levels of service, within each clinical silo (vertical categorization) and for overall ED care (horizontal categorization) for each facility in an area or region (Figures 1 and 2). For any particular categorization scheme, which capabilities define each level are necessarily arbitrary, but should be internally consistent. Furthermore, any such scheme must recognize the disparity of intensive care resources for adult, neonatal, and pediatric patients. ${ }^{14}$ Categorizing each facility in this way allows providers and planners to more easily understand and use what would otherwise be an overwhelming set of data and to readily identify any gaps in services for particular patients within a region. Figure 3 is an example of how categorization might apply to a theoretical 180-bed community

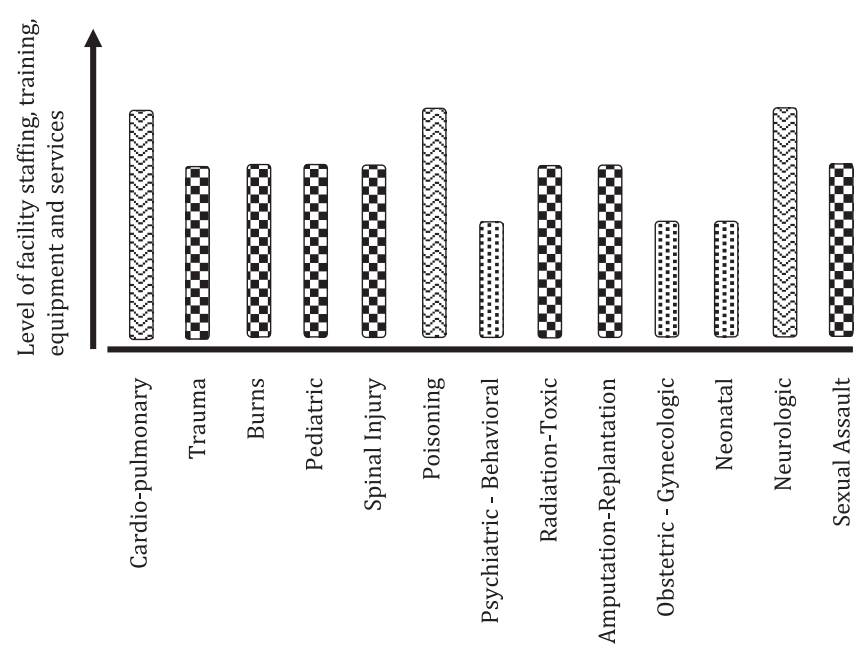

Categorization Silos

\begin{tabular}{|c|c|c|}
\hline \multirow[t]{2}{*}{ 鬼Comprehensive } & 슬 Advanced & Limited \\
\hline & Levels of Service & \\
\hline
\end{tabular}

Figure 3. Categorization of hospital emergency services for a theoretical 180-bed community hospital.

hospital that has no comprehensive level services and limited obstetrical, neonatal, and psychiatric services. For hospitals with limited capacity in one or more vertical silo, the ED would typically stabilize the patient and then initiate coordinated and timely transfer of that patient to the closest appropriate facility.

Within this proposed framework, and in the context of the goals of regionalization, it is important to identify what venues of care would be included. For example, should urgent care centers be considered part of the generalized acute care resources of a community? Would the answer to that question differ in urban areas compared to rural areas, and would the presence of a hospital in close proximity affect the answer? An inclusive approach that allows for the categorization of all relevant emergency care facilities may provide for the most comprehensive coordination of resources.

In an inclusive process, there are both physical and cognitive assets. Physical assets include facilities, such as hospitals, urgent care centers, and both hospital and stand-alone EDs. Cognitive assets include the skills and knowledge of specially trained providers, including those who can offer advice regarding patient care management, and potentially assess and treat via telemedicine or other consultative methods. ${ }^{16}$ These cognitive assets could be represented by critical care specialists, toxicologists, neurosurgeons, neonatologists, emergency physicians, and others. The organized linkage of cognitive assets and physical assets around related clinical conditions typically creates vertical silos like those shown in Figure 1. Designation also involves this kind of linkage in ways that may be characterized by even more tightly integrated resources for high-risk, timesensitive clinical conditions like major trauma, stroke, or STEMI. Telemedical linkages for diagnostic and consultative services could allow a facility to meet criteria for categorization at a higher level for some clinical 
silos and might even allow a facility to achieve designation as a specialized receiving and referral center in limited circumstances. For example, a telemetric neuroradiologist might provide after-hours services for a designated stroke care center.

Designation is an important component of regionalization that is focused on patients who experience a particular medical emergency and who are likely to benefit from the consolidation of intensive care resources. Typically the process involves one or more facilities in a community or region applying for designation as a "center of excellence" for the care of these selected patients. These facilities are then surveyed to determine if capacity, capability, and performance criteria have been or can be met, using quantitative or qualitative criteria, often dependent on self-reported information or data collected for other purposes. The assessment and designation process may entail funding such as from federal or state governments. Designation also requires consideration of the community's or region's needs and the benefits or disadvantages of limiting the number of facilities to be granted exclusive or preferred prehospital receiving or referral status.

Accreditation, as distinguished from designation, looks at the delivery of care and the available personnel and equipment for a wider variety of emergency and nonemergency clinical conditions. It is often identified by third parties like insurance companies, government, and other payers as a necessary step toward ensuring quality, and as a result may be coupled to reimbursement decisions. Accreditation is frequently specified for only a set amount of time before expiring, and therefore requires continuous review to be maintained. It is also not portable to other related parts or locations within the organization being accredited. The process is similar to designation and often times involves site visits and specifically collected and reported data, but generally does not involve the granting of a franchise as a preferred EMS receiving or referral facility.

Categorization and designation are important components of the regionalization of emergency care, but they are not the only elements of the regionalization process. Development and implementation of prehospital triage and transport protocols and policies, identification of service gaps and alternative resources, implementation of interfacility transfer agreements and transport mechanisms, use of off-site cognitive assets through telemetry or remote videoconferencing, and regional disaster planning also play important roles in the regionalization of emergency care. However, the inventory and assessment of existing resources that is completed in the categorization process is an important, if not essential, step in planning a successful, outcome-oriented, and costeffective regionalized emergency care system.

\section{CHALLENGES TO IMPLEMENTATION AND OPPORTUNITIES FOR RESEARCHERS}

There are many potential challenges to categorization, designation, and the development of a regionalized approach to emergency care. These challenges bring with them opportunities for researchers to study categorization and regionalization in terms of design, process, utility, and outcomes, beginning with the basic definitions themselves (Table 1). As the definitions and criteria for these care and planning tools are being formally established, they will need to be subjected to consensus and validation. There are a number of questions and policy issues that can be studied, including the utility of evidence-based categorization criteria, the use of categorization of hospital emergency care to derive prehospital triage protocols, the reliability of self-survey and self-reporting for categorization, the necessity for site survey verification, and the potential for enhanced disaster-preparedness and response.

Additional challenges that research may help resolve include economic and political barriers related to the cost structure of health care delivery and cultural and social barriers related to medical education and physician labor. Some care facilities may be, or may perceive that they may be, financially disadvantaged by a system of categorization and regionalization. Many centers have invested or plan to invest in more specialized and expensive care capacities and capabilities, which overlap with those of neighboring facilities. Categorization, designation, and regionalization can improve transpar-

\section{Table 1}

Research Opportunities in Categorization, Designation, and Regionalization of Emergency Care

\begin{tabular}{ll} 
Category & \multicolumn{1}{c}{ Research Opportunity } \\
\hline Design & - Validation of categorization, designation, and regionalization definitions and criteria. \\
Process & - Replicability of evidence-based standards to categorization and designation criteria. \\
& - Necessity for, and effectiveness of, site survey validation in categorization and designation. \\
- Effect of telemetric services on regionalization, patient transfers, and cost of care. & - Use of categorization in derivation of prehospital triage protocols. \\
- Use of categorization to identify community and regional resource deficits. \\
- Effect of categorization and regionalization on disaster-preparedness and response. \\
- Risks and benefits of patient self-referral to EDs based on categorization and designation. \\
- Implications for medical education and training. \\
- Identification of financial incentives that may promote or discourage regionalization. \\
- Effect of categorization and designation on patient flow, distribution, and hospital financials. \\
- Effect of regionalization on cost of care and outcomes for various clinical silos. \\
- Effect of designation on cost of care and outcomes for specific clinical conditions. \\
- Effect of categorization, designation, and regionalization on resident procedural experience.
\end{tabular}


ency for health care providers and health planners by providing information about staffing, training, and unique vertical capacity, but may also result in changes to patient flow within communities that could have financial consequences for these facilities. The risks and benefits of patients accessing and using categorization information are unclear. This situation could result in unnecessary self-referral to facilities with higher levels of service, disruption of the continuity of care, overcrowded facilities, and additional costs. It could be particularly problematic for smaller hospitals that are near referral centers with more extensive capabilities. In addition, the opportunities for cooperation and collaboration are often limited by legal and political constraints such as antitrust and Stark laws. Thus, transparency concerning categorization, designation, and the development of regionalization can be anticipated to encounter political and economic barriers similar to what historically occurred during trauma center development.

In addition, the telemetric or telephonic connectivity of cognitive assets beyond the geographical location of a physical asset allows for regionalization of care, but may also complicate or exacerbate problems of payment and reimbursement for these services. However, this type of regionalization could lessen the need to transfer patients and thereby diminish the potential economic loss the transferring facility incurs while also reducing overall health care costs. The history of poison center development provides an example of a knowledge-based resource that has proven to decrease the need for patients to be referred to medical facilities through the provision of information and remote management assistance to providers and the public. $^{17,18}$

Incentives that allow for win-win solutions by protecting referral facilities and incorporating them into the regionalized network of care without damaging their economic viability would be important to system development. Research that could identify what those incentives might be and how they could be financed would be helpful in policy development. It is likely that solutions would vary depending upon unique characteristics of a region and its providers, as well as the specific conditions and patient characteristics. The current trend toward hospitals that specialize in cardiac, orthopedic, cancer, or pediatric care has implications for categorization, designation, and regionalized emergency care systems and will also require analysis. There is some evidence to suggest that hospitals and physicians that focus on particular specialized areas of care and develop expertise and experience based on a higher volume of practice may have better outcomes. ${ }^{19}$ The costs and benefits of the application of this expertise to a regionalized system of care will require research specific to each clinical scenario and population being served.

In addition to concerns about competition, experience, quality, and cost, there are numerous other challenges. One of these involves the effect on the education of residents and medical students. Current requirements by most residency review committees involve enumeration of procedural experience. This may negatively affect hospitals that sponsor a training program but are unable to provide experience in a certain procedure because patients are being referred to another facility as part of a regionalization system of care. Research into the implications for medical education and quality of care provided by residency graduates will be needed as categorization and designation of facilities and programs proceed.

Other barriers include the culture of physicians and hospitals that has encouraged increasing specialization, and the development of unique services that make coordination and collaboration difficult between physicians with related but different areas of expertise. In such situations, recognition of the contributions that each specialist can offer and a sharing of revenues may reduce conflict. Analysis of successful models may contribute to continued development of these collaborative services.

Finally, hospitals and their physician staff do not always work in concert or perceive themselves as having unified interests. For categorization, designation, and regionalization to function successfully, hospital facility resources and provider resources must be coordinated toward a common purpose. Incentives and payment systems that reward such behavior could be helpful. Investigations that identify which incentives work, and under what conditions these could be applied, would be valuable to a research agenda on regionalization and categorization.

\section{SUMMARY}

Categorization can be a valuable and perhaps necessary first step in the regionalization of acute care networks. Models of categorization are currently in development and will require evaluation and optimization to ensure that they are achieving their intended goals. Ultimately, categorization, designation, and regionalization will likely evolve in diverse ways in different communities and geographic areas, although there may be potential advantages to the use of a nationally standardized categorization scheme and criteria, especially when it comes to planning for disasters. The development of categorization, designation, and regionalization will provide an excellent opportunity to study these changes through comparative effectiveness research. The definitions, conceptual framework, and discussion of anticipated future challenges and barriers provided in this article can help guide the design and analysis of the costs and benefits of creating a system of categorization and regionalization of emergency care.

The authors acknowledge the breakout session participants from the 2010 Academic Emergency Medicine consensus conference, "Beyond Regionalization: Integrated Networks of Emergency Care."

\section{References}

1. MacKenzie EJ, Rivara FP, Jurkovich GJ, et al. A national evaluation of the effect of trauma-center care on mortality. N Engl J Med. 2006; 354:36678. 
2. MacKenzie EJ, Hoyt DB, Sacra JC, et al. National inventory of hospital trauma centers. JAMA. 2003; 289:1515-22.

3. Schwamm LH, Pancioli A, Acker JE, et al. Recommendations for the establishment of stroke systems of care: recommendations from the American Stroke Association's task force on the development of stroke systems. Stroke. 2005; 36:690-703.

4. LaMonte MP, Bahouth MN, Magder LS, et al. A regional system of stroke care provides thrombolytic outcomes comparable with the NINDS stroke trial. Ann Emerg Med. 2009; 54:319-27.

5. Henry TD, Sharkey SW, Burke MN, et al. A regional system to provide timely access to percutaneous coronary intervention for ST-elevation myocardial infarction. Circulation. 2007; 116:721-8.

6. Nallamothu BK, Bates ER, Wang Y, Bradley EH, Krumholz HM. Driving times and distances to hospitals with percutaneous coronary intervention in the United States: implications for prehospital triage of patients with ST-elevation myocardial infarction. Circulation. 2006; 113:1189-95.

7. Rumack BH, Ford P, Sbarbaro J, Bryson P, Winokur M. Regionalization of poison centers-a rational role model. Clin Toxicol. 1978; 12:367-75.

8. Giffin S, Heard SE. Budget cuts and U.S. poison centers-regional challenges create a nationwide problem. Clin Toxicol. 2009; 47:790-1.

9. Rosenblatt RA, Macfarlane A, Dawson AJ, Cartlidge $\mathrm{PH}$, Larson EH, Hart LG. The regionalization of perinatal care in Wales and Washington State. Am J Public Health. 1996; 86:1011-15.

10. Heath B, Salerno R, Hopkins A, Hertzig J, Caputo M. Pediatric critical care telemedicine in rural underserved emergency departments. Pediatr Crit Care Med. 2009; 10:588-91.
11. Kahn JM, Linde-Zwirble WT, Wunsch $H$, et al. Potential value of regionalized intensive care for mechanically ventilated medical patients. Am J Respir Crit Care Med. 2008; 177:285-91.

12. Kahn JM, Branas CC, Schwab CW, Asch DA. Regionalization of medical critical care: what can we learn from the trauma experience? Crit Care Med. 2008; 36:3085-8

13. Carr BG, Branas CC, Metlay JP, Sullivan AF, Camargo CA Jr. Access to emergency care in the United States. Ann Emerg Med. 2009; 54:261-9.

14. Gausche-Hill M, Schmitz C, Lewis RJ. Pediatric preparedness of US emergency departments: a 2003 survey. Pediatrics. 2007; 120:1229-37.

15. Wiebe RA, Scott SM. Categorization and regionalization of emergency departments caring for children. Clin Pediatr Emerg Med. 1999; 1:45-53.

16. Hersh W, Hickam D, Severance S, Dana T, Krages KP, Helfand M. Diagnosis, access and outcomes: update of a systematic review of telemedicine services. J Telemed Telecare. 2006; 12(Suppl 2):331.

17. Harrison DL, Draugalis JR, Slack MK, Langley PC. Cost-effectiveness of regional poison control centers. Arch Intern Med. 1996; 156:2601-8.

18. Miller TR, Lestina DC. Costs of poisoning in the United States and savings from poison control centers: a benefit-cost analysis. Ann Emerg Med. 1997; 29:239-45.

19. Jollis JG, Peterson ED, DeLong ER, et al. The relation between the volume of coronary angioplasty procedures at hospitals treating medicare beneficiaries and short-term mortality. N Engl J Med. 1994; 331:1625-9.

\section{Academic Emergency Medicine News on FACEBOOK (on SAEM's website)}

Please be sure to regularly frequent and follow many activities of the journal on SAEM's Facebook. Comments on articles are featured there, as well as journal announcements. Another way to keep up to date with the latest information relevant to Academic Emergency Medicine, as well as other emergency medicine topics, happenings, etc! 